\title{
Electrical Polarization in AIN/GaN Nanodisks Measured by Momentum-Resolved 4D Scanning Transmission Electron Microscopy
}

\author{
Knut Müller-Caspary, ${ }^{1,2,}{ }^{*}$ Tim Grieb, ${ }^{3,4}$ Jan Müßener, ${ }^{3}$ Nicolas Gauquelin, ${ }^{2}$ Pascal Hille, ${ }^{3}$ Jörg Schörmann, ${ }^{5}$ \\ Johan Verbeeck, ${ }^{2}$ Sandra Van Aert, ${ }^{2}$ Martin Eickhoff, ${ }^{3}$ and Andreas Rosenauer, ${ }^{3,4}$ \\ ${ }^{1}$ Ernst-Ruska-Centre for Microscopy and Spectroscopy with Electrons, Forschungszentrum Jülich, 52425 Jülich, Germany \\ ${ }^{2}$ EMAT, University of Antwerp, Groenenborgerlaan 171, 2020 Antwerp, Belgium \\ ${ }^{3}$ IFP, University of Bremen, Otto-Hahn-Allee 1, 28359 Bremen, Germany \\ ${ }^{4}$ MAPEX Center for Materials and Processes, University of Bremen, Bibliothekstraße 1, 28359 Bremen, Germany \\ ${ }^{5}$ I. Physikalisches Institut, Justus-Liebig University Gießen, Heinrich-Buff-Ring 16, 35392 Gießen, Germany
}

(Received 13 November 2018; published 11 March 2019)

\begin{abstract}
We report the mapping of polarization-induced internal electric fields in AlN/GaN nanowire heterostructures at unit cell resolution as a key for the correlation of optical and structural phenomena in semiconductor optoelectronics. Momentum-resolved aberration-corrected scanning transmission electron microscopy is employed as a new imaging mode that simultaneously provides four-dimensional data in real and reciprocal space. We demonstrate how internal mesoscale and atomic electric fields can be separated in an experiment, which is verified by comprehensive dynamical simulations of multiple electron scattering. A mean difference of $5.3 \pm 1.5 \mathrm{MV} / \mathrm{cm}$ is found for the polarization-induced electric fields in AlN and GaN, being in accordance with dedicated simulations and photoluminescence measurements in previous publications.
\end{abstract}

DOI: 10.1103/PhysRevLett.122.106102

After the rise of nitride-based semiconductor research nearly three decades ago [1], the investigation of polarization-induced internal electric fields in wurtzite-type heterostructures has rapidly become a major focus in both applied and fundamental physics. GaN-, AlN- or InN-based (compound) crystals exhibit both a spontaneous and a piezoelectric polarization along the [0001] direction for which the magnitudes depend, e.g., on chemical composition or layer thickness in heterostructures. In light emitting devices [2-4], the quantum-confined Stark effect was found responsible for decreased recombination rates due to the spatial separation of the electron and hole wave functions. Although polarization-induced electric fields $[5,6]$ hamper the functionality of many devices [7-9], their experimental quantification with high spatial resolution has not yet been achieved.

Here, we demonstrate the comprehensive characterization of the local polarization in nitride-based axial nanowire heterostructures containing a multistack of AlN/GaN segments at unit cell resolution. To this end, we use momentum-resolved scanning transmission electron microscopy (STEM) as a new imaging mode in which diffraction patterns are recorded on an ultrafast camera of the latest generation [10] in an aberration-corrected STEM. Whereas electric field measurements down to the subatomic scale were demonstrated recently [11-14] in an ultrathin specimen, their separation from the much weaker polarizationinduced electric fields is achieved here for $110 \mathrm{~nm}$ thick wurtzite AlN/GaN nanowires. Field magnitudes of $5.3 \pm 1.5 \mathrm{MV} / \mathrm{cm}$ are found, which are in agreement with photoluminescence data and further interpreted via quantitative modeling using the NEXTNANO ${ }^{3}$ software $[15,16]$, as well as dynamical electron scattering simulations.

Figure 1(a) shows a schematic of the $\mathrm{N}$-polar nanowires grown along the $[000 \overline{1}]$ direction $[6,17]$. The central part of the nanowires contains 40 alternating GaN/AlN segments, as experimentally confirmed by energy-dispersive $\mathrm{x}$-ray spectroscopy data presented in Fig. 1(b), which also shows the epitaxial AlN shell around the wire. STEM experiments were performed at the as-grown wires so that the electrically active specimen thickness equaled the wire diameter along the incident beam direction $[11 \overline{2} 0]$, which we calculated to $110 \pm 10 \mathrm{~nm}$ from the lateral diameter and the hexagonal wire cross section.

The physical background of our momentum-resolved approach is illustrated with the help of simulations in the [1120] projection in Fig. 1(c). A STEM probe is focused to a diameter of approximately $1 \AA$ and rastered across the specimen, where it interacts with the Coulomb potential shown color coded and as a height profile. At each scan position, a diffraction pattern is recorded, which results in a large 4D dataset, i.e., an array of diffraction patterns shown in gray scale in the middle of Fig. 1(c). The magnified part shown in the yellow box depicts one diffraction pattern in the center, corresponding to one scan point. The dashed lines indicate the borders to diffraction patterns for adjacent scan pixels. Finally, the first moment is calculated in each diffraction pattern $[11,18]$, which has been identified as a robust measure of the average momentum transferred to the 

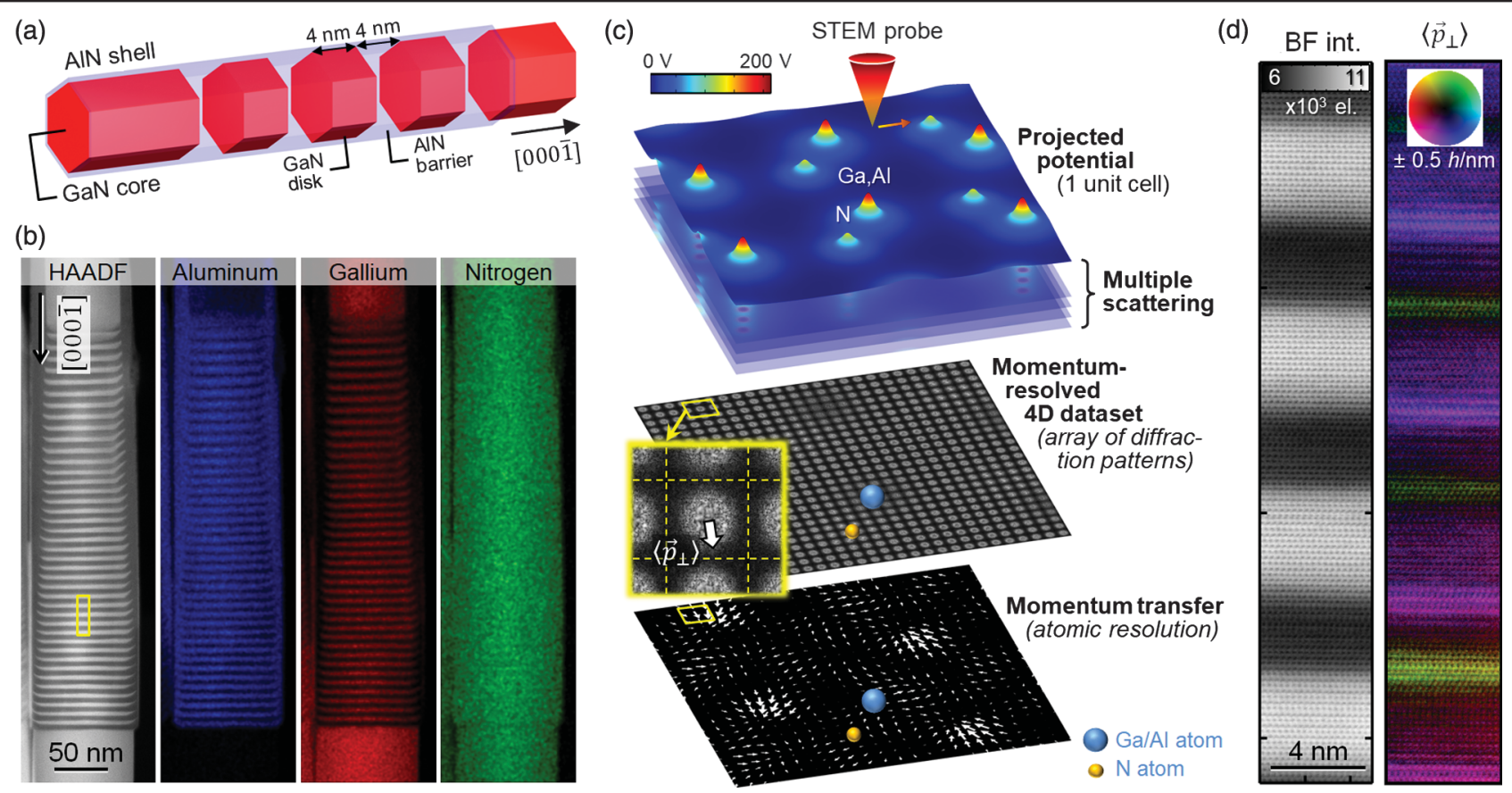

(b)

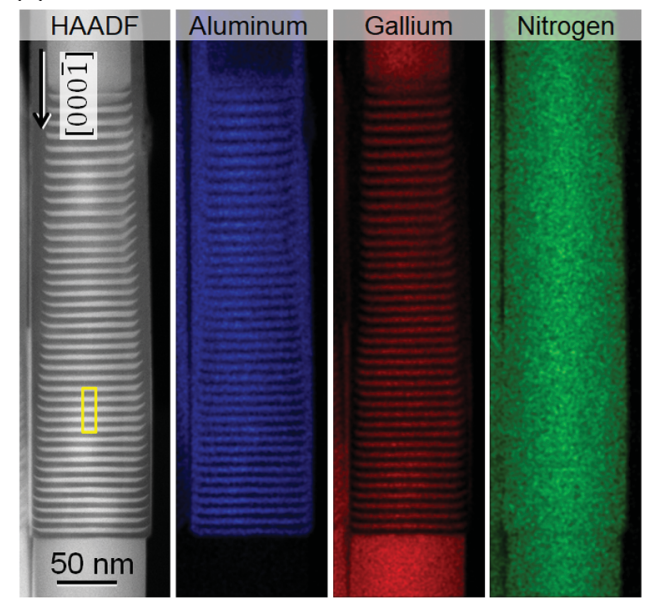

FIG. 1. (a) Schematic of the AlN/GaN wires with $4 \mathrm{~nm}$ layer periodicity and AlN shell. (b) Energy-dispersive X-ray (EDX) spectroscopy and Z-contrast scanning transmission electron microscopy (STEM) image, showing the sequence of pure AlN/GaN layers. (c) Momentum-resolved STEM principle. A probe is multiply scattered in the specimen (top). An array of diffraction patterns is recorded sequentially during rastering (center). For each scan position, the momentum transfer can be determined from the first moment of the diffraction pattern (bottom). (d) Map of the bright field (BF; left) intensity (int.) in numbers of electrons (el.) and the momentum transfers (right, color coded) as determined from the four-dimensional (4D) STEM data acquired within the yellow region in Fig. 1(b).

STEM probe by the specimen [11,19], $\left\langle\vec{p}_{\perp}\right\rangle$. This yields the vector field at the bottom.

The kernel of the electron-specimen interaction consists of the scattering by the projected Coulomb potential of an atomically thin slice and the subsequent free propagation to the next slice. In contrast to previous work dealing with atomic electric fields in an ultrathin specimen [11,14,19], the iteration of this process towards the exit face of the specimen (i.e., multiple scattering) drastically complicates the quantitative analysis. This is easily seen in the momentum transfers at the bottom of Fig. 1(c), in which atomic sites do not at all resemble sinks of momentum transfer.

To solve this problem as to the quantification of polarization-induced fields, we assume that, within one unit cell, the total electric field $\vec{E}(\vec{r})$ can be written as a sum of a constant field $\vec{E}_{c}$ and the strongly varying electric field $\vec{E}_{\text {at }}(\vec{r})$, owing to the atoms. $\vec{E}_{c}$ contains polarizationinduced electric fields or gradients of the mean inner potential, for example. Recalling the application of Ehrenfest's theorem [11,19], the expectation values of the momentum and the electric field are related by

$-\frac{v}{e \Delta z}\left\langle\vec{p}_{\perp}\right\rangle=\frac{1}{\Delta z}\langle\vec{E}(\vec{r})\rangle_{\text {proj }}=\vec{E}_{c}+\frac{1}{\Delta z} \int_{0}^{\Delta z}\left\langle\vec{E}_{\mathrm{at}}(\vec{r})\right\rangle d z$ with $e$ as the elementary charge, $v$ as the electron velocity, and $\Delta z$ as the specimen thickness. The brackets $\langle\ldots\rangle$ denote quantum mechanical expectation values. Equation (1) shows that it is possible to measure $\vec{E}_{c}$ directly in case the momentum transfer caused by the atomic electric fields becomes negligible as compared to $\vec{E}_{c}$, which can be achieved in two ways. First, the electron beam broadens inside the specimen, and hence experiences an intrinsic averaging over atomic-scale variations of $\vec{E}_{\text {at }}(\vec{r})$. Second, we average diffraction patterns [20] or $\left\langle\vec{p}_{\perp}\right\rangle$ over one crystal unit cell or, which yielded the same result, over one Voronoi cell around a $\mathrm{Ga}(\mathrm{Al})-\mathrm{N}$ dumbbell.

The atomically resolved measurement of $\left\langle\vec{p}_{\perp}\right\rangle$ is shown color coded in Fig. 1(d) next to the virtual bright field image. The data were acquired in a part of the yellow region in Fig. 1(b). It consists of 396800 diffraction patterns recorded in a $1600 \times 248$ STEM scan. Both long-range and atomic-scale variations are observed simultaneously, ranging up to $0.5 h / \mathrm{nm}^{-1}$ with the $h$ Planck's constant.

Averaging the momentum transfers in Fig. 1(d) in each Voronoi cell, as depicted in Fig. 2(a), yields $\left\langle\vec{p}_{\perp}\right\rangle^{\mathrm{UC}}$ plotted in Fig. 2(b) separately for the components in [1100] and in the growth direction $[000 \overline{1}]$. Multiplication by $-v / e$ according to Eq. (1) provides the Voronoi cell average of $\langle\vec{E}\rangle$, which equals $\vec{E}_{c} \Delta z$ in Eq. (1) plus a small error 


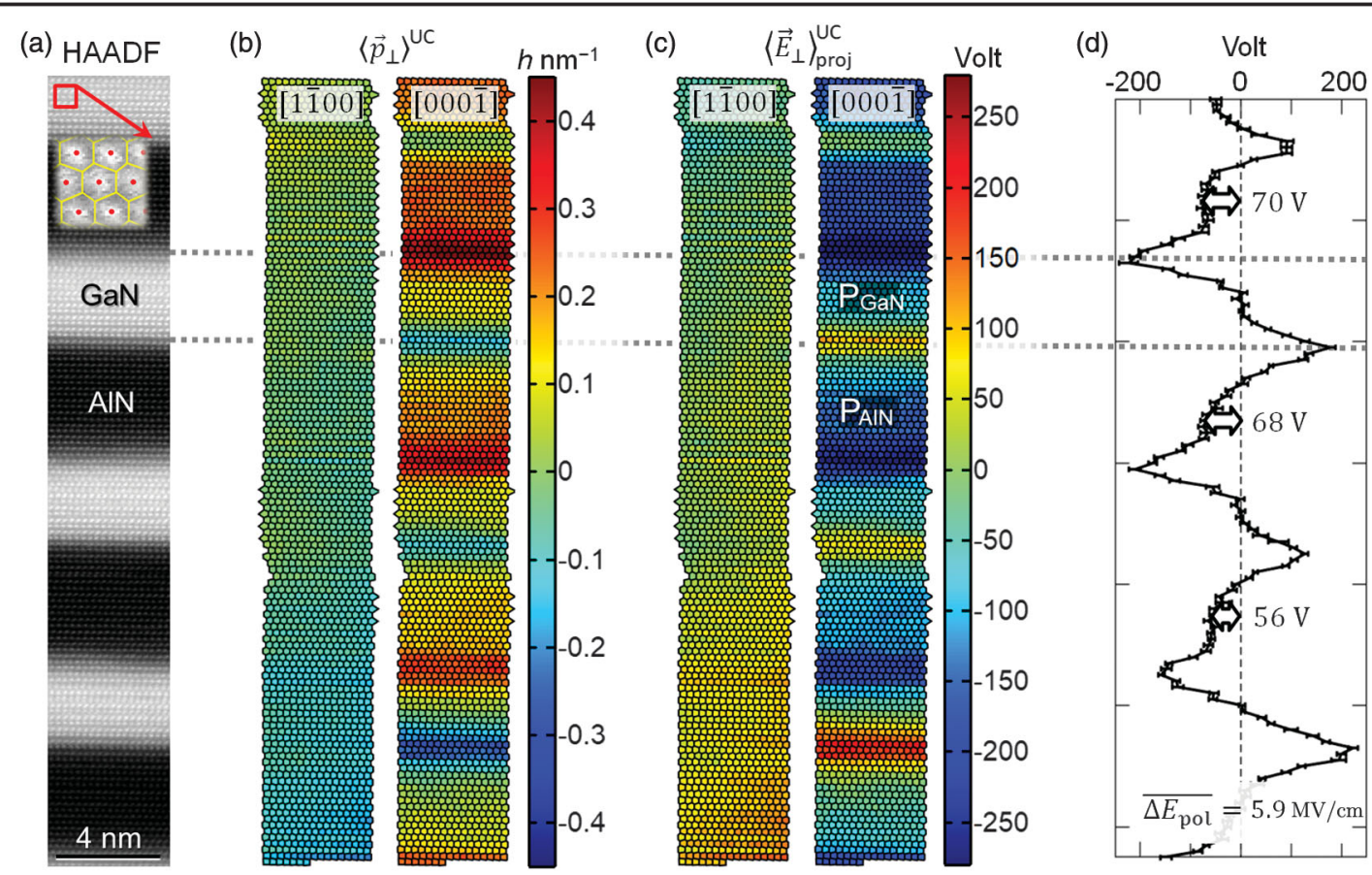

FIG. 2. (a) A high-angle annular dark field (HAADF) STEM image acquired simultaneously with the 4D STEM data is used to calculate the Voronoi diagram. (b) Momentum transfers averaged over the colors separated into the [1 $\overline{1} 00]$ and the $[000 \overline{1}]$ components. (c) Voronoi-cell-averaged electric field projection calculated directly from Fig. 2(b). (d) Line profile along growth direction [0001] after subtraction of a linear slope (black) fitted to the GaN regions. Arrows indicate the electric field differences between AlN and GaN.

$$
\delta=\frac{1}{\Delta z} \overline{\int_{0}^{\Delta z}\left\langle\vec{E}_{\mathrm{at}}(\vec{r})\right\rangle d z,}
$$

where the bar indicates the Voronoi cell average. The influence of $\delta$ will be examined below.

As there are no polarization-induced electric fields in the nonpolar [1 100$]$ direction, $\left\langle\vec{p}_{\perp}\right\rangle_{[1 \overline{1} 00]}^{\mathrm{UC}}$ and $\left\langle\vec{E}_{\perp}\right\rangle_{[1 \overline{1} 00]}^{\mathrm{UC}}$ are almost zero everywhere with a negligible slope due to, e.g., a small thickness gradient. However, $\left\langle\vec{p}_{\perp}\right\rangle_{[000 \overline{1}]}^{\mathrm{UC}}$ and $\left\langle\vec{E}_{\perp}\right\rangle_{[000 \overline{1}]}^{\mathrm{UC}}$ take values of up to $\pm 0.4 \mathrm{~h} / \mathrm{nm}^{-1}$ and $\pm 280 \mathrm{~V}$ at interfaces that mainly originate from different mean inner potentials of GaN and AlN. A closer look at regions well inside the GaN and AlN layers reveals plateaus in which the projected electric field is almost constant. These plateaus exhibit different levels for $\left\langle\vec{E}_{\perp}\right\rangle_{[000 \overline{1}]}^{\mathrm{UC}}$ in AlN and GaN, as indicated exemplarily for two plateaus $P_{\mathrm{AlN}}$ and $P_{\mathrm{GaN}}$ in Fig. 2(c). Here, a difference of approximately $68 \mathrm{~V}$ is found, originating from the material-dependent polarization field. With the specimen thickness of $\Delta z=110 \mathrm{~nm}$, this corresponds to an electric field of approximately $6.4 \mathrm{MV} / \mathrm{cm}$. The plateaus of 70,68 , and $56 \mathrm{~V}$ are more

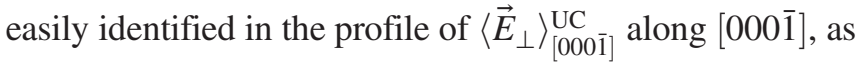
shown in Fig. 2(d). Because we are only interested in the difference of the electric field in $\mathrm{GaN}$ and $\mathrm{AlN}$, a linear fit to the centers of the GaN layers in the profile was subtracted. Assuming the specimen thickness to be in the range of $110 \pm 10 \mathrm{~nm}$, we find an average polarizationinduced electric field of $5.9 \pm 0.5 \mathrm{MV} \mathrm{cm}^{-1}$. Moreover, it is worth noting that our analysis focuses on the center of the wire as marked in Fig. 1(b). Applying the method to areas interfacing with vacuum could in general be equally instructive so as to detect electric stray fields originating from surface dipole charge layers, causing both the $[000 \overline{1}]$ and the [1100] components of the electric field to be nonzero. However, this is hindered by the AlN shell in the present case.

To gain deeper insight, we model the electric field profile in Fig. 2(d) by assuming that a gradient of the chemical composition $c$ causes both a gradient of the mean inner potential and of the polarization-induced electric field. Regions with constant composition contribute to polarization-induced fields only. Hence, we can define a model

$$
\Delta E_{\text {model }}=\alpha \frac{d}{d x} c(x)+\beta c(x)+\gamma,
$$

where $x$ denotes the dependency along the growth direction; and $\alpha, \beta$, and $\gamma$ are fit parameters. The composition profile $c(x)$ is derived from the high-angle annular dark field STEM signal [21] in Fig. 3(a), and Fig. 3(b) shows its (negative) gradient. Comparison with the experiment in Fig. 3(d) reveals that Fig. 3(b) yields the correct behavior at interfaces, whereas the profile in Fig. 3(a) is responsible for the different plateau levels inside AlN and GaN. Finally, the 
(a) STEM intensity profile, $\propto \mathrm{c}(x)$

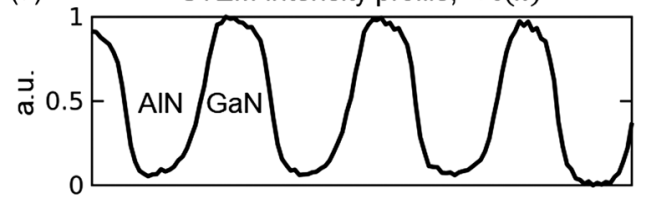

(b)

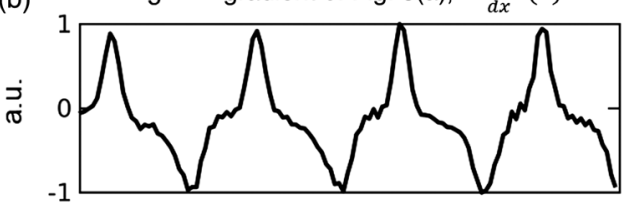

(c) Model fit $\Delta E_{\text {model }}=\alpha \cdot \frac{d}{d x} c(x)+\beta \cdot c(x)+\gamma$

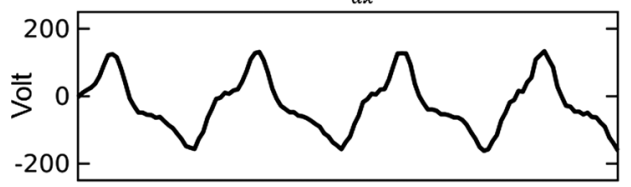

(d) Exp. profile $\left\langle\vec{E}_{\perp}\right\rangle_{\text {proj }}^{\mathrm{UC}},[000 \overline{1}]$ component

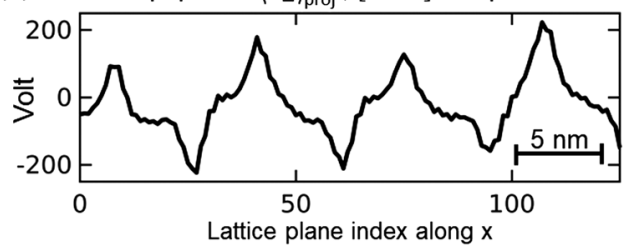

FIG. 3. Modeling the electric field in growth direction. (a) HAADF STEM profile for the region in Fig. 2, considered as being proportional to the chemical composition $c(x)$. (b) Negative gradient of Fig. 3(a). (c) Linear combination of Figs. 3(a) and 3(b) according to Eq. (3), determined by fitting the experimental (Exp.) profile to Fig. (d).

fit result in Fig. 3(c) is in very good agreement with the experimental profile in Fig. 3(d). With $E_{\mathrm{pol}}=\beta \cdot c(x)$, we find $\Delta E_{\mathrm{pol}}=5.3 \mathrm{MV} / \mathrm{cm}$ for the difference of the polarization-induced electric field in $\mathrm{AlN}$ and $\mathrm{GaN}$, whereas an error of $\pm 0.5 \mathrm{MV} / \mathrm{cm}$ is to be assumed due to an inaccuracy of the thickness measurement of $\pm 10 \mathrm{~nm}$.

It is instructive to integrate the component arising from the gradient in Eq. (3) along $x$ over an AlN/GaN interface, which yields a potential of $2.1 \mathrm{~V}$. This is already close to literature values for the mean inner potential differences of $\mathrm{GaN}$ and AlN $[23,24]$ around $1 \mathrm{~V}$, but further studies on dynamical scattering at interfaces ought to be performed for full quantification. So far, our method yields both the polarization within nanometer-sized layers and the correct qualitative behaviour at interfaces, with either of them being absent in previous reports [25-27].

We now return to the systematic error $\delta$ in Eq. (2), which can arise from the violation of inversion symmetry in wurtzite nitrides [19]. To this end, we neglect polarizationinduced electric fields in the simulation [28], and we calculate the unit-cell-averaged momentum transfer for both AlN and GaN up to a wire thickness of $150 \mathrm{~nm}$. This yields the momentum transfer that arises solely due to the crystal structure, which could misleadingly be interpreted as being caused by internal fields. The difference between the thickness dependence of $\delta$ for GaN and AlN is plotted in Fig. 4(a). $\delta$ takes large magnitudes of up to $10-20 \mathrm{MV} / \mathrm{cm}$ for specimen thicknesses lower than $30 \mathrm{~nm}$, which would preclude the quantification of polarization-induced fields. However, $\delta$ suffers a hyperbolic attenuation with specimen thickness owing to Eq. (2), leading to tolerable errors in the thickness range above $100 \mathrm{~nm}$, like in the present experiments. The red bar marks a maximum error of $\pm 1 \mathrm{MV} / \mathrm{cm}$ for the nanowire under study.

To conclude, we measure an average difference of $5.3 \mathrm{MV} / \mathrm{cm}$ for the polarization-induced electric fields in AlN and GaN, with uncertainties of $\pm 0.5 \mathrm{MV} / \mathrm{cm}$ and $\pm 1 \mathrm{MV} / \mathrm{cm}$ due to the inaccurately known specimen thickness and dynamical scattering in non-centrosymmetric crystals. This is in good agreement with recent measurements employing bias-controlled photoluminescence [6], which predicted electric polarization fields larger than $3.5 \mathrm{MV} / \mathrm{cm}$ for nanowires with multiple pairs of $4 \mathrm{~nm}$ thick GaN disks and $>3 \mathrm{~nm}$ thick AlN barriers.

Furthermore, NEXTNANO ${ }^{3}$ simulations $[15,16]$ were performed that take the actual 3D wire geometry, including the AlN shell, $N$ polarity, and surface Fermi-level pinning (a)

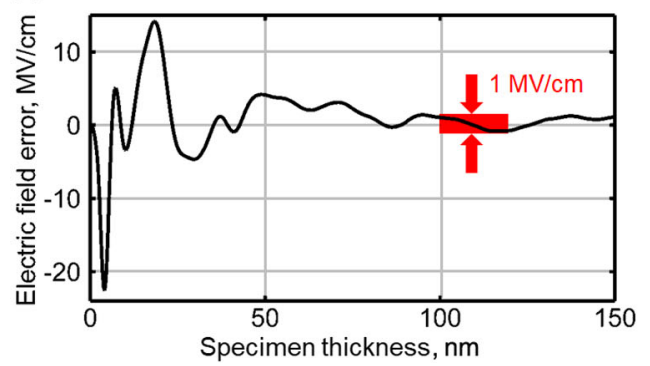

(b)

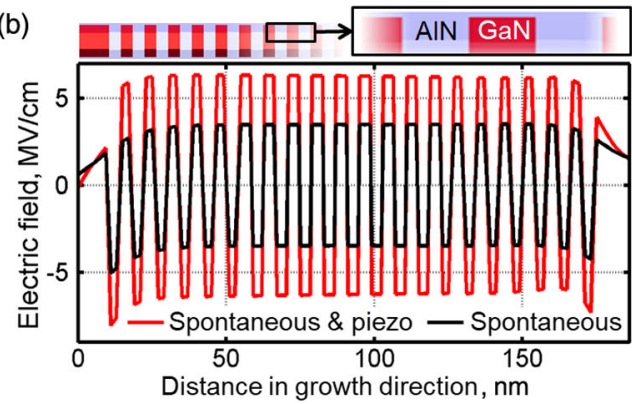

(c)

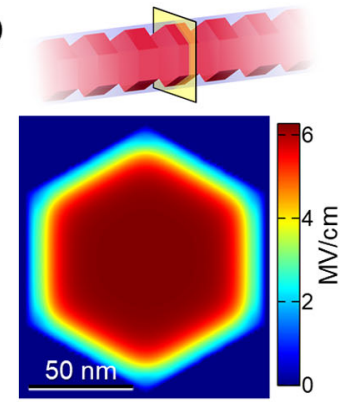

FIG. 4. (a) Thickness-dependent simulation of the systematic error $\delta$ in Eq. (2), arising from the lack of inversion symmetry in wurtzite GaN/AIN. It amounts to $1 \mathrm{MV} / \mathrm{cm}$ for the present wire. (b) NEXTNANO ${ }^{3}$ simulation of the electric field ([0001] component) assuming the actual three-dimensional (3D) wire geometry and fully strained (red) or fully relaxed (black) layers. (c) NEXTNANO ${ }^{3}$ simulation of the electric field $([000 \overline{1}]$ component) across a cross-sectional slice centered in a GaN segment. 
at midgap into account [6]. By solving Poisson's equation, the electrostatic potential and field were computed, and profiles along the nanowire's central axis were extracted $[17,29]$ as shown in Fig. 4(b). Strain energy was minimized as described earlier [17], assuming fully coherent interfaces. As the degree of the plastic relaxation is unknown, Fig. 4(b) shows two asymptotic cases with (red) and without (black) piezoelectric fields, respectively. For the two situations, mean polarization field differences of $6.8 \mathrm{MV} / \mathrm{cm}$ (black) and $12.5 \mathrm{MV} / \mathrm{cm}$ (red) are obtained. In addition, we extracted a cross-sectional slice through a GaN segment as depicted in Fig. 4(c). Obviously, the electric field magnitude decreases gradually towards the wire edges. Averaging the field in the direction of the STEM beam yields approximately $85 \%$ of the field at the central axis. Thus, the NEXTNANO ${ }^{3}$ simulations finally predict fields between 5.8 and $10.6 \mathrm{MV} / \mathrm{cm}$ for a fully relaxed wire and a fully strained wire, respectively.

The difference to the measured value of $5.3 \mathrm{MV} / \mathrm{cm}$ can be partially explained by (plastic) strain relaxation as observed previously [17]. Similar discrepancies have been reported by Strak et al. [30], where ab initio calculations gave polarization-induced fields of $7.7 \mathrm{MV} / \mathrm{cm}$, whereas photoluminescence results yielded only $4.5 \mathrm{MV} / \mathrm{cm}$ and the evaluation of the Berry phase provided field strengths between 5 and $6.7 \mathrm{MV} / \mathrm{cm}$ [30,31]. In addition, the measurement of internal electric fields is affected by mobile screening charges due to (unintentional) doping [32] or charges generated by the electron beam [33]. Concerning GaN/AlN heterostructures, our results are rather consistent with fields of $6.9 \mathrm{MV} / \mathrm{cm}$ from electron holography reports [25] that, however, dealt with a different layer geometry and did not elucidate the impact of the mean inner potential.

In summary, we achieved the mapping of polarizationinduced electric fields in III-V nitrides with unit cell resolution by momentum-resolved STEM. This included the separation of atomic electric fields, mean inner potential gradients, and polarization effects. The measured electric field difference of $5.3 \mathrm{MV} / \mathrm{cm}$ in an epitaxially grown $40 \times \mathrm{GaN}(4 \mathrm{~nm}) / \mathrm{AlN}(4 \mathrm{~nm})$ disk sequence is beyond the scope of application of bias-controlled photoluminescence approaches [6], making momentum-resolved STEM a key approach for the electrical characterization of novel nanoelectronic devices in the future.

The authors gratefully acknowledge the help of Natalie Claes for analyzing the EDX data. K. M.-C. acknowledges funding from the Initiative and Network Fund of the Helmholtz Association within the Helmholtz Young Investigator Group moreSTEM under Contract No. VHNG-1317 at Forschungszentrum Jülich in Germany. The direct electron detector (Medipix3, Quantum Detectors) was funded by the Hercules fund from the Flemish Government. N. G. and J. V. acknowledge funding from the Geconcentreerde Onderzoekacties project Solarpaint of the University of Antwerp. T. G. and A. R. acknowledge support from the Deutsche Forschungsgemeinschaft (Germany) under Contract No. RO2057/8-3. This work also received funding from the European Research Council under the European Union's Horizon 2020 research and innovation programme (Contract No. 770887). The authors acknowledge financial support from the Research Foundation Flanders (FWO, Belgium) through project funding (G.0368.15N).

K. M.-C. and T. G. contributed equally to this work and share first-authorship.

*k.mueller-caspary@fz-juelich.de

[1] S. Nakamura, T. Mukai, and M. Senoh, Appl. Phys. Lett. 64, 1687 (1994).

[2] F. Qian, Y. Li, S. Gradečak, H.-G. Park, Y. Dong, Y. Ding, Z. L. Wang, and C. M. Lieber, Nat. Mater. 7, 701 (2008).

[3] A. G. Sarwar, S. D. Carnevale, F. Yang, T. F. Kent, J. J. Jamison, D. W. McComb, and R. C. Myers, Small 11, 5402 (2015).

[4] A. T. M. G. Sarwar, B. J. May, M. F. Chisholm, G. J. Duscher, and R. C. Myers, Nanoscale 8, 8024 (2016).

[5] H. Masui, H. Asamizu, T. Melo, H. Yamada, K. Iso, S. C. Cruz, S. Nakamura, and S. P. DenBaars, J. Phys. D 42, 135106 (2009).

[6] J. Müßener, P. Hille, T. Grieb, J. Schörmann, J. Teubert, E. Monroy, A. Rosenauer, and M. Eickhoff, ACS Nano 11, 8758 (2017).

[7] L. Rigutti, M. Tchernycheva, A. D. L. Bugallo, G. Jacopin, F. H. Julien, R. Songmuang, E. Monroy, S. T. Chou, Y. T. Lin, P. H. Tseng, L. W. Tu, F. Fortuna, L. Zagonel, M. Kociak, and O. Stephan, Phys. Status Solidi (A) 207, 1323 (2010).

[8] J. Teubert, P. Becker, F. Furtmayr, and M. Eickhoff, Nanotechnology 22, 275505 (2011).

[9] G. Capuzzo, D. Kysylychyn, R. Adhikari, T. Li, B. Faina, A. Tarazaga Martín-Luengo, and A. Bonanni, Sci. Rep. 7, 42697 (2017).

[10] R. Plackett, I. Horswell, E. N. Gimenez, J. Marchal, D. Omar, and N. Tartoni, J. Instrum. 8, C01038 (2013).

[11] K. Müller, F. F. Krause, A. Beche, M. Schowalter, V. Galioit, S. Löffler, J. Verbeeck, J. Zweck, P. Schattschneider, and A. Rosenauer, Nat. Commun. 5, 5653 (2014).

[12] F. Winkler, J. Barthel, A. H. Tavabi, S. Borghardt, B. E. Kardynal, and R. E. Dunin-Borkowski, Phys. Rev. Lett. 120, 156101 (2018).

[13] N. Shibata, S. D. Findlay, Y. Kohno, H. Sawada, Y. Kondo, and Y. Ikuhara, Nat. Phys. 8, 611 (2012).

[14] K. Müller-Caspary, M. Duchamp, M. Rösner, V. Migunov, F. Winkler, H. Yang, M. Huth, R. Ritz, M. Simson, S. Ihle, H. Soltau, T. Wehling, R. E. Dunin-Borkowski, S. Van Aert, and A. Rosenauer, Phys. Rev. B 98, 121408 (2018).

[15] A. Trellakis, T. Zibold, T. Andlauer, S. Birner, R. K. Smith, R. Morschl, and P. Vogl, J. Comput. Electron. 5, 285 (2006). 
[16] S. Birner, T. Zibold, T. Andlauer, T. Kubis, M. Sabathil, A. Trellakis, and P. Vogl, IEEE Trans. Electron Devices 54, 2137 (2007).

[17] F. Furtmayr, J. Teubert, P. Becker, S. Conesa-Boj, J. R. Morante, A. Chernikov, S. Schäfer, S. Chatterjee, J. Arbiol, and M. Eickhoff, Phys. Rev. B 84, 205303 (2011).

[18] E. M. Waddell and J. N. Chapman, Optik (Stuttgart) 54, 83 (1979).

[19] K. Müller-Caspary, F. F. Krause, T. Grieb, S. Löffler, M. Schowalter, A. Béché, V. Galioit, D. Marquardt, J. Zweck, P. Schattschneider, J. Verbeeck, and A. Rosenauer, Ultramicroscopy 178, 62 (2017).

[20] J. M. LeBeau, S. D. Findlay, L. J. Allen, and S. Stemmer, Ultramicroscopy 110, 118 (2010).

[21] See Supplemental Material at http://link.aps.org/ supplemental/10.1103/PhysRevLett.122.106102, for the calibration of the STEM detector, which includes Ref. [22].

[22] J. M. LeBeau and S. Stemmer, Ultramicroscopy 108, 1653 (2008).

[23] M. Schowalter, A. Rosenauer, D. Lamoen, P. Kruse, and D. Gerthsen, Appl. Phys. Lett. 88, 232108 (2006).

[24] A. S. W. Wong, G. W. Ho, R. E. Dunin-Borkowski, T. Kasama, R. A. Oliver, P. M. Costa, and C. J. Humphreys, MRS Proc. 892, 0892 (2005).

[25] L. Zhou, D. A. Cullen, D. J. Smith, M. R. McCartney, A. Mouti, M. Gonschorek, E. Feltin, J. F. Carlin, and N. Grandjean, Appl. Phys. Lett. 94, 121909 (2009).
[26] M. den Hertog, R. Songmuang, F. Gonzalez-Posada, and E. Monroy, Jpn. J. Appl. Phys. 52, 11NG01 (2013).

[27] D. Carvalho, K. Müller-Caspary, M. Schowalter, T. Grieb, T. Mehrtens, A. Rosenauer, T. Ben, R. García, A. RedondoCubero, K. Lorenz, B. Daudin, and F. M. Morales, Sci. Rep. 6, 28459 (2016).

[28] A. Rosenauer and M. Schowalter, STEMSIM-a new software tool for simulation of STEM HAADF Z-contrast imaging, in Microscopy of Semiconducting Materials (Springer, Dordrecht, 2007), pp. 170-172.

[29] J. Müßener, J. Teubert, P. Hille, M. Schäfer, J. Schörmann, M. de la Mata, J. Arbiol, and M. Eickhoff, Nano Lett. 14, 5118 (2014).

[30] P. Strak, P. Kempisty, K. Sakowski, A. Kaminska, D. Jankowski, K. P. Korona, K. Sobczak, J. Borysiuk, M. Beeler, E. Grzanka, E. Monroy, and S. Krukowski, AIP Adv. 7, 015027 (2017).

[31] Y. Kotsar, B. Doisneau, E. Bellet-Amalric, A. Das, E. Sarigiannidou, and E. Monroy, J. Appl. Phys. 110, 033501 (2011).

[32] P. Hille, J. Müßener, P. Becker, M. de la Mata, N. Rosemann, C. Magén, J. Arbiol, J. Teubert, S. Chatterjee, J. Schörmann, and M. Eickhoff, Appl. Phys. Lett. 104, 102104 (2014).

[33] J. B. Park, T. Niermann, D. Berger, A. Knauer, I. Koslow, M. Weyers, M. Kneissl, and M. Lehmann, Appl. Phys. Lett. 105, 094102 (2014). 


\section{Supplementary Information for: Electrical polarisation in AlN/GaN nanodiscs measured by momentum-resolved 4D-STEM}

\section{EXPERIMENTAL DETAILS}

For the experimental work, the probe-corrected FEI XAnt-EM transmission electron microscope at the EMAT institute in Antwerp (Belgium) was used. The microscope was operated at $120 \mathrm{kV}$ with a semi-convergence angle of $20.7 \mathrm{mrad}$ of the STEM probe.

Residual aberrations were measured from Zemlin tableaus as follows:

\begin{tabular}{l|c|c}
\hline Aberration & Value & Angle \\
\hline 2-fold astigmatism (A1) & $3.8 \mathrm{~nm}$ & $-116.8^{\circ}$ \\
3-fold astigmatism (A2) & $44.6 \mathrm{~nm}$ & $-51.1^{\circ}$ \\
Axial coma (B2) & $24.4 \mathrm{~nm}$ & $152.8^{\circ}$ \\
Spherical aberration (C 3$)$ & $-1.6 \mu \mathrm{m}$ & n.a. \\
4-fold astigmatism (A3) & $2.2 \mu \mathrm{m}$ & $-139.5^{\circ}$ \\
Star aberration (S3) & $823.9 \mathrm{~nm}$ & $15.4^{\circ}$ \\
5-fold astigmatism (A4) & $21.6 \mu \mathrm{m}$ & $-10.4^{\circ}$ \\
\hline
\end{tabular}

The ultrafast direct electron detection system[1] Medipix 3 Merlin for EM (Quantum Detectors) was operated in Single Pixel Mode (SPM) with a sensor bias of $120 \mathrm{~V}$ and a lower threshold of $60 \mathrm{keV}$ to eliminate double-counting of electrons with $120 \mathrm{keV}$ energy. The pixel depth was 6 bit and the acquisition was synchronised with the STEM dwell time of $820 \mu$ s per scan pixel.

A Fischione Model 3000 annular detector was used to record the high-angle annular dark field signal simulta- neously with the 4D STEM data. The inner acceptance angle of the detector was $56 \mathrm{mrad}$, determined from the shadow of the detector hole on the Medipix sensor and a known diffraction pattern. The outer angle of the detector was determined by measuring both the inner and outer radius of the sensitive detector area by scanning the STEM beam over the detector in imaging mode [2] (detector scan). With the ratio of both radii and the aforementioned inner angle, an outer acceptance angle of $195 \mathrm{mrad}$ is calculated.
[1] R. Plackett, I. Horswell, E. N. Gimenez, J. Marchal, D. Omar, and N. Tartoni, Journal of Instrumentation 8, C01038 (2013).
[2] J. M. LeBeau and S. Stemmer, Ultramicroscopy 108, 1653 (2008). 